\title{
Friend recommendation based on the Luscher color theory: Twitter use case
}

\begin{abstract}
At the area of information technology, social networks are becoming an unavoidable part of the Internet usage, due to their facilities for users as well as the benefits to the providers. However, the popularity and thereby, the success of social networks depends highly on number of the network members. This in turn, depends on considerations for several criteria such as networking and number of networks. On the other hand, it is believed that suggestion of appropriate friends to members performed by an effective recommender system can lead to suitable content ranking and consequently, impact the growth of the network in significant sense. The current paper introduces a novel method of recommending celebrities in a social network based on genetic algorithm and the concept of color psychology. The proposed method is applied to Twitter social network as case study, through which the cost function of the user is first optimized to achieve the ideal weights, and the celebrities are then ranked based on the specified parameters as follower and following counts, background color and description. The system is tested using real data of celebrities and number of 100 users with identical parameters. The results evidence the closest recommendation in terms of affordable recommendation error rates as low as $7.6 \%$ based on the psychological data validation.
\end{abstract}

Keyword: Recommender system; Social network; Twitter; Max Luscher color theory; Genetic algorithm 